\title{
Elimination of malaria due to Plasmodium vivax in central part of the People's Republic of China: analysis and prediction based on modelling
}

\author{
Zhao Chen ${ }^{1}$, Liang Shi ${ }^{2}$, Xiao-Nong Zhou ${ }^{3}$, Zhi-Gui Xia ${ }^{3}$, Robert Bergquist ${ }^{4}$, Qing-Wu Jiang ${ }^{1}$ \\ ${ }^{1}$ School of Public Health, Fudan University, Shanghai, People's Republic of China; ${ }^{2}$ Jiangsu Institute of Parasitic \\ Diseases, Key Laboratory of Parasitic Disease Control and Prevention (Ministry of Health), Jiangsu Provincial \\ Key Laboratory of Parasite Molecular Biology, Wuxi, People's Republic of China; ${ }^{3}$ National Institute of \\ Parasitic Diseases, Chinese Center for Disease Control and Prevention, Shanghai, People's Republic of China; \\ ${ }^{4}$ Ingerod, Brastad, Sweden
}

\begin{abstract}
Five provinces in central People's Republic of China (P.R. China) have successfully reduced the burden of malaria due to Plasmodium vivax in the last 7 years. The results of the Action Plan of China Malaria Elimination (APCME) that commenced in 2010 are analysed against the background of the progress reached by the national malaria control programme (NMEP) that was launched in 2006. We examined the epidemiological changes in the number of autochthonous cases over time and discuss the feasibility of achieving the goal of malaria elimination by 2020 . There was a total decline of 34,320 malaria cases between 2006 and 2012 arriving at an average annual incidence of 0.04 per 10,000 people by 2012. At the same time, the number of counties reporting autochthonous cases declined from 290 to 19. Spatial autocorrelation and Bayesian modelling were used to evaluate the datasets and predict the spatio-temporal pattern in the near future. The former approach showed that spatial clusters of P. vivax malaria existed in the study region during the study period, while the risk prediction map generated by the Bayesian model indicates that only sporadic malaria cases will appear during in the future. The results suggest that the initial NMEP approach and the follow-up APCME strategy have played a key role in reducing the threat of malaria in central P.R. China. However, to achieve the goal of malaria elimination by the end of the current decade, intervention plans must be adjusted with attention paid to those endemic counties still at risk according to the prediction map.
\end{abstract}

Keywords: malaria, Plasmodium vivax, elimination, epidemiology, Bayesian modelling, spatial autocorrelation, prediction, People's Republic of China.

\section{Introduction}

Both Plasmodium falciparum and P. vivax were historically prevalent in the People's Republic of China (P.R. China). The former was successfully eliminated together with a significant reduction of $P$. vivax cases in the early 1990s (Sleigh et al., 1998; Lu et al., 2014). The global malaria incidence decreased by $17 \%$ between 2000 and 2010 (Feachem et al., 2010; WHO, 2012) and the trend has been similar in P.R. China. However, P. vivax remains a challenge (Gething et al., 2012 ) in the central parts of the country (Tang, 2000). The number of cases here reportedly reached 22 million, accounting for $91.2 \%$ of the total number of all cases in P.R. China in 1970 (Huang et al., 2011). Since

\footnotetext{
Corresponding author:

Qing-Wu Jiang

School of Public Health, Fudan University

20025 Shanghai, People's Republic of China

Tel./Fax +86 21 5423-7435

E-mail: jiangqw@fudan.edu.cn
}

then, the number of cases decreased dramatically (Xu et al., 1994; Li et al., 1999). However, by the end of the $20^{\text {th }}$ century, malaria due to P. vivax re-emerged and the situation was again dire in the provinces along the Huang-Huai River (Yellow River), especially in the Henan and Anhui provinces (Sleigh et al., 1998; Zhou et al., 2005). A total of 34,933 malaria cases were reported in 2006, the highest number of malaria cases so far in the new millennium (Huang et al., 2011), resulting in the immediate launch of the national malaria control programme (NMEP). Good results were achieved and the progress has been followed up by the Action Plan of the China Malaria Elimination (APCME) taking over in 2010 with the explicit aim of malaria elimination. The process was accelerated by shifting the control approach from "reduce incidence in the highly endemic areas" to "block malaria transmission in each epidemic unit" (Yin et al., 2013; Zheng et al., 2013). The endemic counties were classified into four types based on the local malaria incidence in the period of 2006-2008. Type I consisted of counties with local infections above 1/10,000 during 3 consecutive 
years; type II counties were those with local infections during the latest 3 years including at least one year with an incidence less than 1/10,000 (but above 0); type III counties were defined as those with no local infections reported during 3 years; and type IV were those declared completely non-endemic for malaria.

Control and management of infection sources were strengthened in all counties with enhanced targeting of "hotspots" located in type I and type II counties. The new approach is based on controlling infection sources and implementing long-term treatment during the malaria resting phase (Yin et al., 2013) consisting of the following objectives:

(i) elimination in all "malaria-epidemic areas" (MEA) in type III counties by 2015 ;

(ii) elimination of the disease in all type I and type II counties except for some border regions in Yunnan province by 2015;

(iii) drop of the general autochthonous malaria incidence to $1 / 10,000$ in all type I counties, including those in the Yunnan border regions by 2015;

(iv) no new autochthonous malaria cases detected in the whole country from 2017; followed by

(v) the realisation of national malaria elimination by 2020.

This approach aims to achieve malaria elimination with a definition of no autochthonous cases for 3 consecutive years in the whole country by 2020 . The new control strategy includes surveillance activities and the "1-3-7" approach, which entails reporting malaria cases within one day, confirmation and investigation carried out before the end of 3 days followed by prevention of further transmission within 7 days with targets set to guide and monitor follow-up each case (Bergquist et al., 2014; Cao et al., 2014). Being a vector-borne disease correlated with environmental and socioeconomic factors (Tang, 2000; Yang et al., 2012; Wang et al., 2014), malaria typically produces spatial and temporal clusters (Bi et al., 2013; Noor et al., 2014). The development of new tools of control as well as improvement of existing approaches for the rapid and accurate identification of all active cases are critical to the endeavour of malaria elimination. The institution of better control measures is facilitated by spatial epidemiology, which provides an understanding of the distribution patterns of many diseases leading to the identification of risk factors and targeting of specific problems (Elliott and Wartenberg, 2004; Ostfeld et al., 2005; Noor et al., 2014). This is achieved by application of statistical approaches, such as spatial autocorrelation (Reid et al., 2010; Yang et al., 2013) and Bayesian geostatistical modelling (Yang et al., 2008; Alegana et al., 2013).

In this study, we analyse the malaria status in central P.R. China and present evidence for the changing epidemiological situation after the launch of the elimination strategy. We draw attention to existing risk factors, predict the future incidence of malaria using Bayesian geostatistical modelling and provide suggestions aimed at improving the strategies and interventions for eliminating malaria in P.R. China.

\section{Materials and methods}

\section{Study area}

The centre of P.R. China includes the area between the Yangtze River and the Yellow River (the vast Huang-Huai plain), which also includes some hillocks and hilly areas. The study area comprises about $723,000 \mathrm{~km}^{2}$ and consists of the provinces of Jiangsu, Anhui, Henan, Shandong with Hubei in the South. This region is located between latitudes $28^{\circ} 54^{\prime}$ $38^{\circ} 12^{\prime} \mathrm{N}$ and $108^{\circ} 16^{\prime}-122^{\circ} 42^{\prime} \mathrm{E}$ along the HuangHuai River. A population of about 451.37 million is distributed over 605 counties that include 103 meteorological monitoring stations (Fig. 1).

\section{Data collection}

Data on malaria cases at the county level between 2006 and 2012 were collected according to the annual submissions from provincial centres for disease control and prevention (CDCs) and institutes of parasitic disease (IPDs).

The Meteorological Data Sharing Service System (http://www.cdc.cma.gov.cn/) provided the annual average temperature $\left(\mathrm{T}_{\text {avg }}\right)$, the average relative humidity $\left(\mathrm{H}_{\text {avg }}\right)$ and the average rainfall. Since the meteorological stations are not evenly distributed in P.R. China, some of the the local meteorological parameters were estimated through spatial prediction using kriging interpolation with ArcGIS version 10.1 (Bowden et al., 2011).

\section{Statistical analysis}

Data were entered into Microsoft Excel 2010 and then imported into SPSS version 17.0 for analysis. The differences were evaluated using the $\chi^{2}$ method considering P-values $<0.05$ statistically significant. Autochthonous P. vivax malaria cases are referred to in accordance with the incidence rate (per 100,000 people) and the constituent ratio, the latter representing the proportion of cases reported each year in com- 


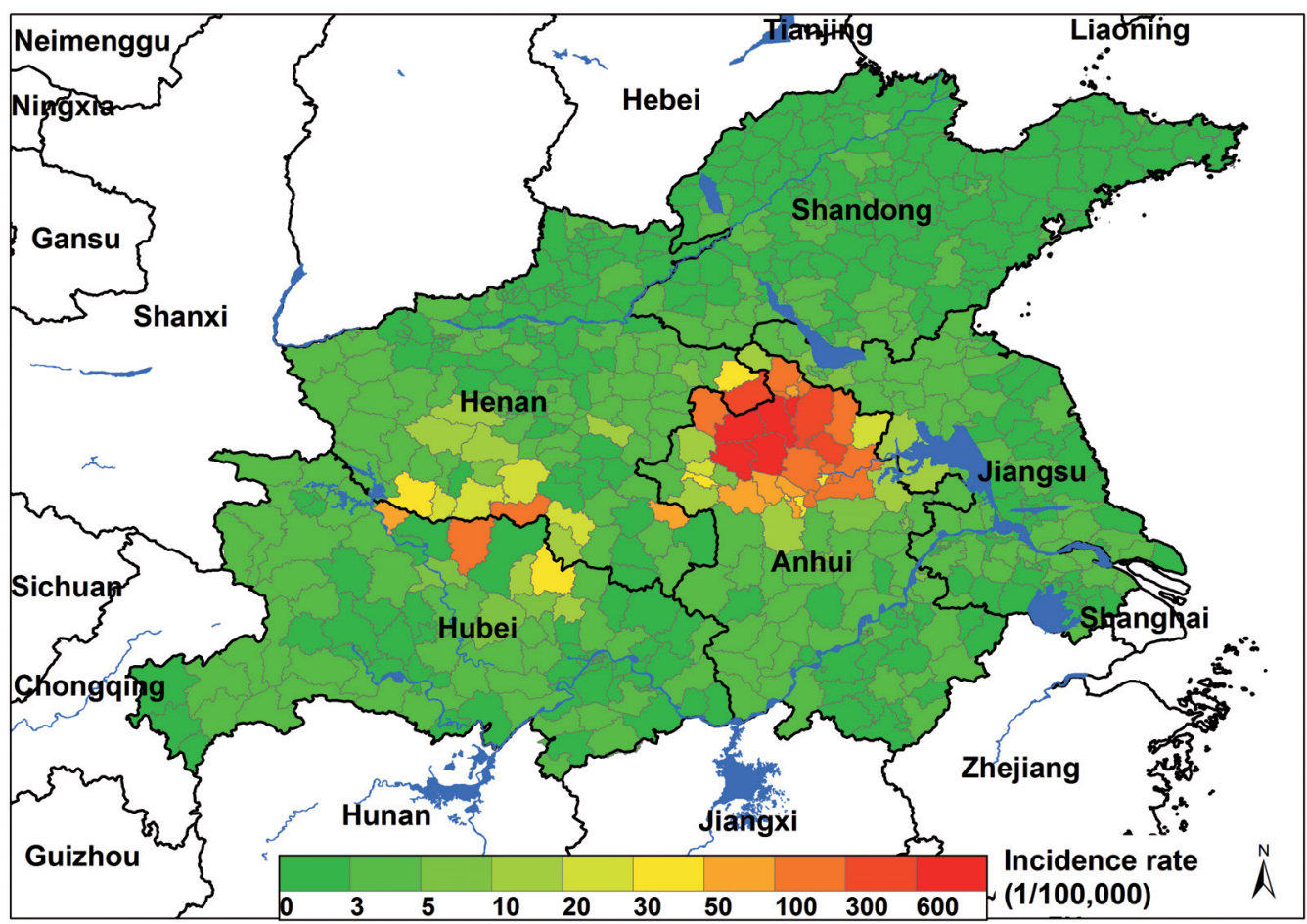

Fig. 1. The distribution and incidence rate of P. vivax malaria in central P.R. China in 2006. Incidence rates expressed as number of cases per 100,000 inhabitants.

parison to the total population (or counties with cases in comparison to all counties). Reported cases were calculated by county and the spatial distribution and temporal malaria trend analysed as described below.

Spatial autocorrelation was used for cluster exploration and $\mathrm{Z}$ score for assessment of the significance of Moran's $I$. The annual differences were then used to explore the spatio-temporal patterns. The distribution of malaria cases by area at the county level was then further examined by Bayesian statistical approaches. The relationship between incidence and environmental characteristics as well as control-related covariates was explored. Bayesian non-spatial and spatio-temporal Poisson models were fitted in WinBUGS (Medical Research Council Biostatistics Unit, Cambridge, United Kingdom). The goodness-of-fit was explored by the deviance information criterion (DIC). Model parameters were estimated using Markov chain Monte Carlo (MCMC) simulation.

Assuming that the observed malaria case counts $\left(O_{i t}\right)$ in county $i$ and year $t$ follow a Poisson distribution, i.e. $O_{i t} \sim$ Poisson $\left(\mu_{i t}\right)$, we introduced covariates, spatial and spatio-temporal effects on the log transformation of $\mu_{i t}$ and developed five models for $\log \left(\mu_{i t}\right)$. In model 1 , neither spatial nor temporal correlation was assumed; in model 2, only county-specific random effects were included; in model 3 , only year-specific random effects were included; in model 4 , county- and year-specific independent random effects were included; and in model 5, spatial correlation evolving over time (space-time interaction) was assumed. Expressed mathematically, the models appear as:

$$
\begin{array}{lr}
\log \left(\mu_{i t}\right)=\log \left(E_{i t}\right)+\alpha+\sum_{k} \beta_{k} X_{i t k} & (\text { model 1) } \\
\log \left(\mu_{i t}\right)=\log \left(E_{i t}\right)+\alpha+\sum_{k} \beta_{k} X_{i t k}+u_{j} & (\text { model 2) } \\
\log \left(\mu_{i t}\right)=\log \left(E_{i t}\right)+\alpha+\sum_{k} \beta_{k} X_{i t k}+v_{t} & (\text { model 3) } \\
\log \left(\mu_{i t}\right)=\log \left(E_{i t}\right)+\alpha+\sum_{k} \beta_{k} X_{i t k}+u_{j}+v_{t} & (\text { model 4) } \\
\log \left(\mu_{i t}\right)=\log \left(E_{i t}\right)+\alpha+\sum_{k} \beta_{k} X_{i t k}+u_{j t} & (\text { model 5) }
\end{array}
$$

In each model, $E_{i t}$ represents the expected malaria case count in county $i$ at time $t$; while $\alpha$ is the intercept, $\beta_{k}$ the regression coefficient and $X_{i t k}$ denotes the covariates including climate data and any particular APCME control activities, e.g. the 1-3-7 approach. The control strategy was set as the dummy variable with the value before $2010=0$ since these control measures were not in effect before that year; otherwise the value $=1$ was used. In models 2,3 and 4 , the variables $u_{j}$ and $v_{t}$ represent county-specific and year-specific random effects, respectively; $u_{j}$ is a random term 
allowing for spatially structured variation in the malaria case dataset, while $v_{t}$ is a random term representing annual variation. Spatial correlation was modelled using county-specific random effects, which were assumed to arise from $r$, a conditional autoregressive model (CAR), which implies that each $\boldsymbol{u}_{j}$ is conditional on the neighbour $u_{m}$ and follows a normal distribution with the mean equal to the average of the neighbouring $u_{m}$ and the variance equal to $\delta_{j}^{2}$ scaled according to the number of the neighbours of village $j$ resulting in the equation:

$$
u_{j} \mid u_{m, j \neq j} \sim N\left(r \sum_{m=1}^{n_{n}} u_{j}, \delta_{j}^{2} / n_{j}\right)
$$

where $r$ quantifies the strength of spatial dependence. Temporal correlation was modelled by annual random effects under the assumption that they follow an autoregressive process (1) with lag 1, assuming that temporal correlation $r$ exists only with the preceding year. In model 4, the spatial correlation between villages is independent of the year. In model $5, u_{j t}$ is the spatiotemporal random effect such that $u_{j t} \sim \operatorname{CAR}\left(r_{t}\right)$ with the parameter $r_{t}$ controls the rate of decrease of spatial correlation with distance in year $t$.

Following a Bayesian model formulation, we adopted vague normal prior distributions for each regression coefficient $\beta_{k}$ and intercept $\alpha$, vague inverse gamma priors for variances and a uniform prior ranging from -1 to +1 for spatial and temporal correlation. One-chain MCMC simulation was used for parameter estimation. The inference of the parameters was based on a burn-in of 15,000 iterations of both chains after the $30,000^{\text {th }}$ iteration.

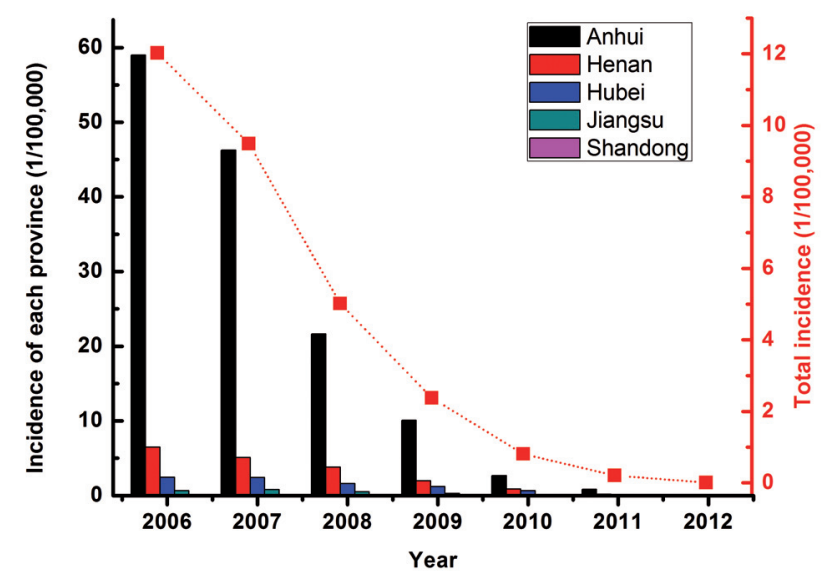

Fig. 2. Annual incidence of P. vivax malaria between 2006 and 2012 in the study area. Incidence per 100,000 with the bars showing the situation in each province and the red line indicating the total incidence in the five provinces together.
Table 1 . The incidence rate of $P$. vivax malaria in central P.R. China at the beginning and end of the study.

\begin{tabular}{lrrrrr}
\hline \multirow{2}{*}{ Province } & \multicolumn{2}{c}{2006} & & \multicolumn{2}{c}{2012} \\
\cline { 2 - 3 } \cline { 5 - 6 } \cline { 5 - 6 } & $\begin{array}{c}\text { Number } \\
\text { of cases }\end{array}$ & $\begin{array}{c}\text { Incidence } \\
\text { per } 100,000\end{array}$ & $\begin{array}{c}\text { Number } \\
\text { of cases }\end{array}$ & $\begin{array}{c}\text { Incidence } \\
\text { per 100,000 }\end{array}$ \\
\hline Anhui & 34,933 & 58.99 & & 30 & 0.05 \\
Henan & 5,030 & 6.48 & & 0 & 0.00 \\
Hubei & 1,259 & 2.46 & & 9 & 0.02 \\
Jiangsu & 453 & 0.66 & & 0 & 0.00 \\
Shandong & 91 & 0.10 & & 0 & 0.00 \\
\hline
\end{tabular}

The calculations were carried out under the assumption that the 2015 climate factors will be similar to those of 2014 and that the APCME control strategy continues to be carried out without change. The predicted number of malaria cases in each county was estimated by the model having the best fit. Based on the estimate of malaria cases for each county, maps were generated to show the prediction distributions of the infection rate evaluating the possibility of malaria elimination in 2015 , because all autochthonous P. vivax malaria would be eliminated in the study region based on APCME.

\section{Results}

\section{General epidemic situation}

A total of 104,904 cases of P. vivax malaria were reported in the period from 2006 to 2012 in central P.R. China. As Figs. 1 and 2 show, most cases were in northern Anhui and in the border area between the Henan and Hubei provinces. The average, annual malaria incidence decreased from $12.02 / 100,000$ to only $0.01 / 100,000$ in the study area from 2006 to

Table 2. Malaria endemicity in the two periods with different control strategies.

\begin{tabular}{lcccccc}
\hline \multirow{2}{*}{ Programme } & \multirow{2}{*}{ Period } & \multicolumn{5}{c}{ Type } \\
\cline { 3 - 7 } & & $\mathrm{I}^{\mathrm{a}}$ & II $^{\mathrm{b}}$ & III $^{\mathrm{c}}$ & IV $^{\mathrm{d}}$ & $\begin{array}{c}\text { No. of } \\
\text { counties }\end{array}$ \\
\hline NMPE & $2006-2008$ & 37 & 364 & 204 & 0 & 605 \\
APCME & $2010-2012$ & 0 & 218 & 387 & 0 & 605 \\
\hline
\end{tabular}

a Local infections detected in 3 consecutive years with the annual incidence rate $\geq 1 / 10,000$; blocal infections detected in the last 3 years with incidence between 0 and 1/10,000 in at least one year; 'no local infections reported during the last 3 years; darea non-endemic for malaria. 


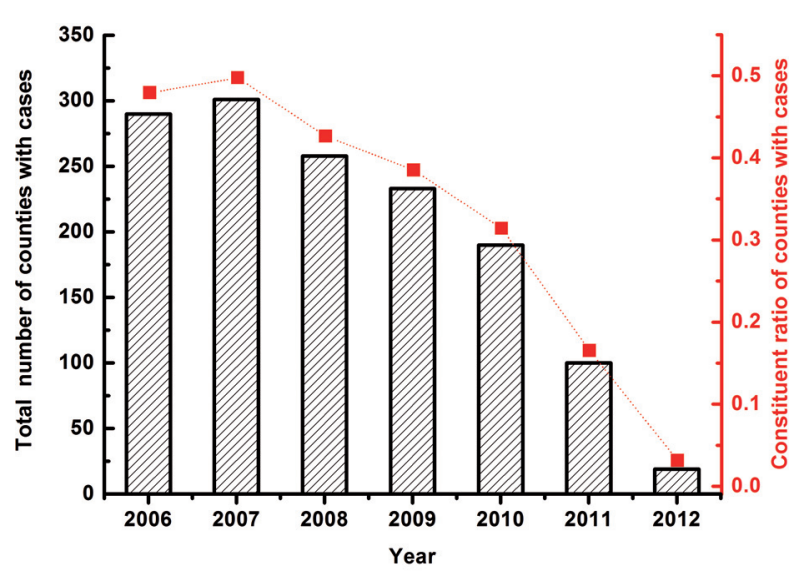

Fig. 3. The number of counties reporting $P$. vivax malaria between 2006 to 2012 in the study area.

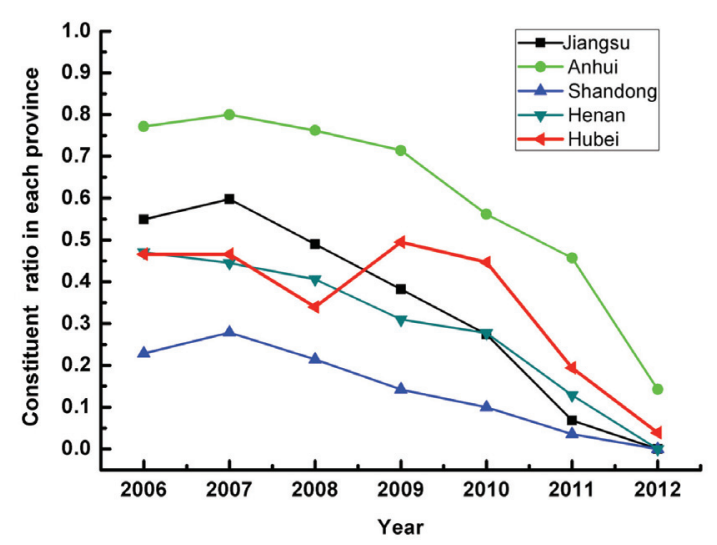

Fig. 4. Counties reporting P. vivax malaria in central P.R. China between 2006 and 2012.

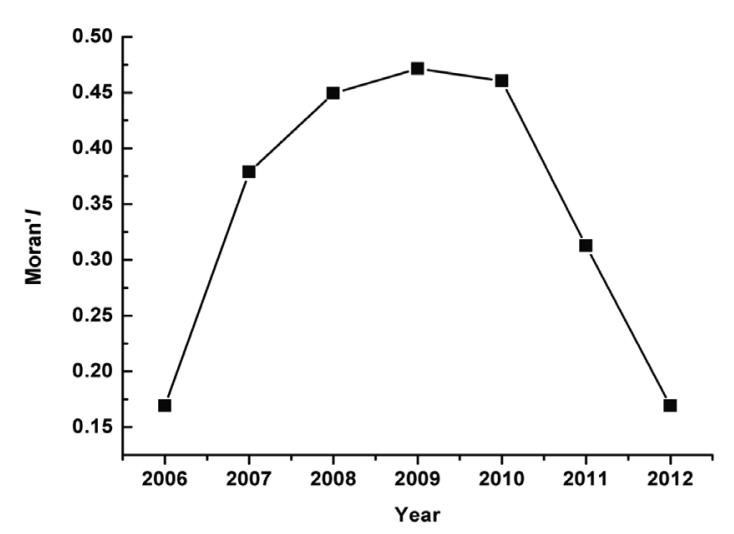

Fig. 5. Variation of Moran's I of P. vivax malaria in central P.R. China between 2006 and 2012.
2012. The incidence dropped in each province with Jiangsu, Henan and Shandong, covering $68 \%$ of the total study population, not reporting any case in 2012 . Meanwhile, the average, annual malaria incidence in Anhui and Hubei decreased to $30(0.05 / 100,000)$ and $9(0.02 / 100,000)$, respectively (Table1).

\section{Variation of endemic counties}

Fig. 3 depicts the strong, general decrease in autochthonous malaria over the study period. Five provinces (290 counties) reported malaria cases in 2006 as compared to only 19 counties $\left(\chi^{2}=211.9\right.$; $\mathrm{P}<0.001)$ in two provinces $(\mathrm{P}=0.003)$ in 2012. However, the situation in Anhui, Jiangsu, Shandong and Hubei provinces was not stable. The number of counties reporting cases in these four provinces was 84 in 2007, 36 in 2008 and 51 in 2009, respectively, which was even higher than in the year 2006 (Fig. 4).

Tables 1 and 2 compare the change in endemic counties with different strategies in different periods. Classification of MEA according to APCME shows that there were 37 type I counties in the period of 2006-2008. No county was re-ranked as type I, but 187 counties shifted from type II to type III in the period 2010-2012. The constituent ratio of types I-IV was statistically significant in two periods, i.e. 2006-2008 and 2009-2012 ( $\left.\chi^{2}=130.3 ; \mathrm{P}<0.001\right)$.

As Fig. 5 and Table 3 show, the spatial autocorrelation analysis of the annualised incidence of malaria in the five provinces showed that the Moran's $I$ was statistically significant from 2006 and 2012 ( $\mathrm{P}<0.01)$. The findings imply that the malaria distribution was generally auto-correlated. The Moran's I increased from 2006 to 2009, decreased in 2010 to dramatically increase afterwards.

Table 3. Moran's I with regard to of $P$. vivax malaria in the five provinces between 2006 and 2012.

\begin{tabular}{lcccc}
\hline Year & Moran's $I$ & Variance & Z-value & P-value \\
\hline 2006 & 0.169 & 0.000279 & 10.22 & $<0.01$ \\
2007 & 0.379 & 0.000265 & 23.38 & $<0.01$ \\
2008 & 0.449 & 0.00028 & 26.93 & $<0.01$ \\
2009 & 0.471 & 0.00029 & 27.77 & $<0.01$ \\
2010 & 0.460 & 0.000298 & 26.76 & $<0.01$ \\
2011 & 0.312 & 0.000299 & 18.17 & $<0.01$ \\
2012 & 0.169 & 0.000279 & 10.22 & $<0.01$ \\
\hline
\end{tabular}


Table 4 summarises the analyses of the Poisson Bayesian models. The DIC values of Model 4, including county- and year-specific independent random effects, was smaller than in the other Bayesian models, suggesting that the spatio-temporal independent Bayesian model had a better fit. This model was consequently used for risk factor analysis and risk map generation. From the spatio-temporal correlation structures of the malaria status in model 4, it was obvious that the temporal correlation was not significant at the $95 \%$ credible intervals (CIs) including 0 . The spatial variation was less than the temporal variation, indicating that the apparent similarity of the number of malaria cases in comparison with the neighbouring counties could be higher than that of the preceding year.

According to Table 4, all correlation coefficients were positive and the difference between the climate factors and malaria prevalence showed a statistical significance at the $95 \% \mathrm{CI}$ including 0 . The APCME control measure variable was negative and was also statistically significant.

Fig. 6 shows the risk distribution based on model predictions with lower risk in green and higher risk in red. The model predictions indicate that most counties should not have any new cases. However, 12 counties in different parts of the study area might still have cases in 2015, in particular in the provinces of Anhui, Henan and Hubei. Anhui province would have the highest estimated infection rate $(0.12 / 100,000)$.

\section{Discussion}

In order to make progress towards malaria elimination, the global malaria map needs to shrink and the application of geospatial sciences play an important role (Clements et al., 2013; Walker et al., 2013; Giardina et al., 2014). Progress achieved thus far in P.R. China supports the possibility that elemination of malaria can be achieved, at least in certain parts of the world, e.g. the re-emergence of malaria was effectively controlled by NMEP. The number of autochthonous cases declined rapidly and the incidence of new cases were less than 1/100,000 in 2012, while the number of counties with reported cases was as low as $3 \%$ $(19 / 605)$ by the end of 2012. However, although the overall incidence declined smoothly, the number of counties with malaria cases increased from 2006 to

Table 4. Estimates of the parameters in the Bayesian models for P. vivax malaria investigated.

\begin{tabular}{|c|c|c|c|c|c|c|}
\hline Factor & & Model 1* & Model 2* & Model 3* & Model 4* & Model 5* \\
\hline Intercept & & $\begin{array}{c}-0.075 \\
(-0.081 ;-0.068)\end{array}$ & $\begin{array}{c}-0.060 \\
(-0.399 ; 0.315)\end{array}$ & $\begin{array}{c}-3.907 \\
(-4.002 ;-3.820)\end{array}$ & $\begin{array}{c}-2.182 \\
(-2.479 ;-1.849)\end{array}$ & $\begin{array}{c}-0.121 \\
(-0.451 ; 0.236)\end{array}$ \\
\hline \multirow[t]{2}{*}{ Control strategy } & No & - & - & - & - & - \\
\hline & Yes & $\begin{array}{c}0.030 \\
(-0.001 ; 0.062)\end{array}$ & $\begin{array}{c}-0.015 \\
(-0.860 ; 0.793)\end{array}$ & $\begin{array}{c}0.367 \\
(0.323 ; 0.412)\end{array}$ & $\begin{array}{c}-1.120 \\
(-1.670 ;-0.492)\end{array}$ & $\begin{array}{c}-0.965 \\
(-1.255 ; 0.325)\end{array}$ \\
\hline Average rainfall & & $\begin{array}{c}0.461 \\
(0.449 ; 0.473)\end{array}$ & $\begin{array}{c}0.466 \\
(0.454 ; 0.477)\end{array}$ & $\begin{array}{c}0.046 \\
(0.025 ; 0.067)\end{array}$ & $\begin{array}{c}0.256 \\
(0.222 ; 0.291)\end{array}$ & $\begin{array}{c}0.429 \\
(0.321 ; 0.810)\end{array}$ \\
\hline Average temperature & & $\begin{array}{c}-0.146 \\
(-0.160 ;-0.131)\end{array}$ & $\begin{array}{c}-0.146 \\
(-0.161 ;-0.131)\end{array}$ & $\begin{array}{c}0.202 \\
(0.067 ; 0.341)\end{array}$ & $\begin{array}{c}0.657 \\
(0.495 ; 0.833)\end{array}$ & $\begin{array}{c}-0.256 \\
(-0.851 ;-0.101)\end{array}$ \\
\hline Average relative humidity & & $\begin{array}{c}0.018 \\
(-0.007 ; 0.043)\end{array}$ & $\begin{array}{c}0.010 \\
(-0.016 ; 0.035)\end{array}$ & $\begin{array}{c}1.088 \\
(0.979 ; 1.202)\end{array}$ & $\begin{array}{c}1.108 \\
(1.003 ; 1.217)\end{array}$ & $\begin{array}{c}0.121 \\
(-0.236 ; 0.614)\end{array}$ \\
\hline Temporal correlation & & - & $\begin{array}{c}0.008 \\
(-0.645 ; 0.625)\end{array}$ & - & $\begin{array}{c}-0.477 \\
(-0.832 ; 0.284)\end{array}$ & - \\
\hline Temporal variation & & - & $\begin{array}{c}3.564 \\
(1.071 ; 8.457)\end{array}$ & - & $\begin{array}{c}0.610 \\
(0.192 ; 1.499)\end{array}$ & - \\
\hline Spatial variation & & - & - & $\begin{array}{c}0.134 \\
(0.115 ; 0.156)\end{array}$ & $\begin{array}{c}0.135 \\
(0.115 ; 0.157)\end{array}$ & - \\
\hline $\mathrm{DIC}^{* *}$ & & $610,644.0$ & $610,612.0$ & $25,163.2$ & $24,410.0$ & $55,123.5$ \\
\hline
\end{tabular}

\footnotetext{
*Posterior median with $(95 \%$ credible interval); **deviance information criterion.
} 


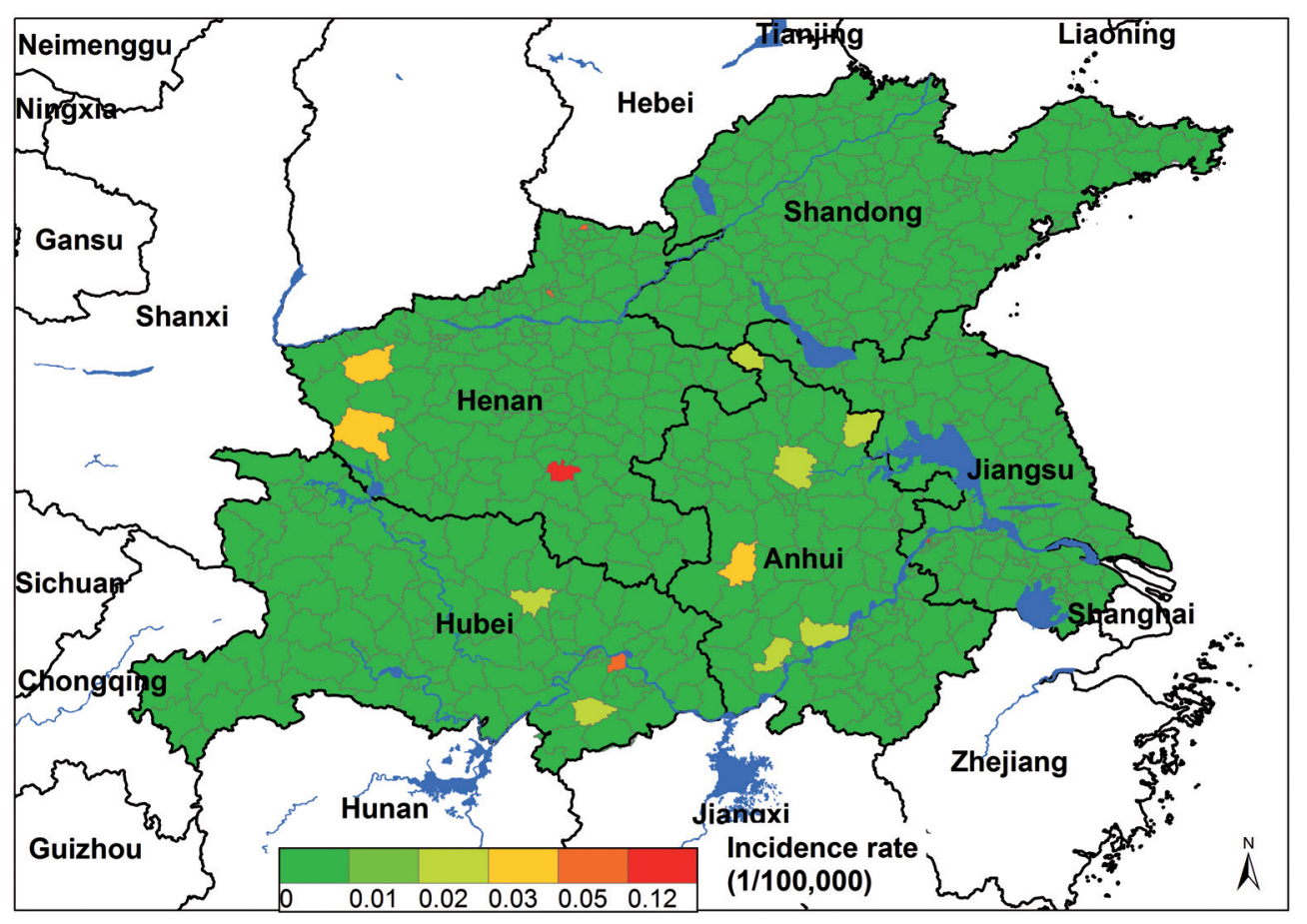

Fig. 6. The predicted incidence rate of P. vivax malaria in central P.R. China in for 2015. The colour spectrum gives the predicted rates per 100,000 people.

2009 in Hubei province to peak in 2009. This may be explained by the local government relaxing existing control measures prematurely or misunderstanding the differences between control phases. Alternatively, it could be a side effect of the improved water quality of Huang-Huai River since this amplified the rice planting area, a situation that expanded the breeding habitats of the Anopheles vector (Huang et al., 2011). It could also have to do with long-term dormant liver stages of P. vivax resulting in relapse of the infection after the end of treatment (Mueller et al., 2009).

The results received after the shift in control approach in 2010 confirm the efficacy of the APCME. As can be seen in Fig. 3, the effectiveness of the new control strategy is shown by the dramatic, continuous drop of the number of autochthonous cases. In correspondence with other studies ( $\mathrm{Bi}$ and Tong, 2014; Diouf et al., 2014; Lu et al., 2014), the 2010-2012 part of the curve drops faster than that of 2006-2009.

Clustering not only reflects an uneven distribution pattern, but also provides clues as to the influencing factors. For example, the spatial autocorrelation analysis techniques used to see whether there was any regional clustering revealed that malaria was far from randomly distributed in the study area; indeed, it peaked in 2009 when the number of cases was clearly concentrated along the Huang-Huai River. Obviously, the high population density and the climate environment were suitable for breeding of the mosquito vector in this case (Zhou et al., 2010; Bai et al., 2013), a strong hint of the close correlation between malaria and the local environmental and socioeconomic conditions. In 2006, a few years after the launch of APCME, the value of Moran's I had decreased considerably indicating that the epidemiological status had reached a level typical for counties with a low incidence of $P$. vivax malaria. This analysis supports the conclusion that APCME has had a good effect.

The Bayesian model has improved our understanding of the contribution of the APCME strategy. The model with random effects independent of specific spatial and temporal influence, selected according to the DIC value to investigate environmental and ecosocial determinants (Alemu et al., 2014; Arab et al., 2014), may thus be useful for decision-making and policy setting. We established that the key climate factors for malaria infection varied in the provinces studied, a finding fully supported by other, recent communications (Caminade et al., 2014; Weiss et al., 2014). The goal of malaria elimination could be reached as early as 2018, but the strategy is faced by severe challenges. Firstly, the main vectors (Anopheles sinensis and An.anthropophagus) still exist in central P.R. China, so transmission is still possible. Secondly, 
research challenges in low-prevalence settings, such as the role of asymptomatic infections, remain (Stresman et al., 2012). Thirdly, the sharp reduction of the need for professional treatment teams as a result of the aim shifting from control to elimination, could undermine long-term prevention (Cotter et al., 2013).

Limitations of the current study must also be recognised: neither specific data regarding the different species of vectors involved, nor socioeconomic variables were taken into account in the prediction model. However, a number of urgent needs were confirmed. Firstly, management improvements are required in counties at risk, as discussed by Musa et al. (2012). Secondly, strengthened monitoring of local malaria hotspots is pivotal (Zheng et al., 2013). Thirdly, implementation of real-time surveillance and effective response system targeting vulnerable environments and populations must be explosed (Zhou et al., 2013). Fourthly, capacity building aimed at sustaining current treatment teams are necessary (Tambo et al., 2012).

\section{Acknowledgements}

We wish to thank the staff members in charge of malaria elimination in the national institutes of parasitic diseases and Jiangsu Institute of Parasitic Diseases in P.R. China. This project was supported by National Natural Science Foundation of China (grant no. 81273192). Xiao-Nong Zhou was supported by the National S\&T Major Program (grant no. 2012ZX10004220) and the China UK Global Health Support Programme (grant no. GHSP-CS-OP3).

\section{References}

Alegana VA, Atkinson PM, Wright JA, Kamwi R, Uusiku P, Katokele S, Snow RW, Noor AM, 2013. Estimation of malaria incidence in northern Namibia in 2009 using Bayesian conditional-autoregressive spatial-temporal models. Spat Spatiotemporal Epidemiol 7, 25-36.

Alemu K, Worku A, Berhane Y, Kumie A, 2014. Spatiotemporal clusters of malaria cases at village level, northwest Ethiopia. Malar J 13, 223.

Arab A, Jackson MC, Kongoli C, 2014. Modelling the effects of weather and climate on malaria distributions in West Africa. Malar J 13, 126.

Bai L, Morton LC, Liu Q, 2013. Climate change and mosquitoborne diseases in China: a review. Global Health 9, 10.

Bergquist R, Yang GJ, Knopp S, Utzinger J, Tanner M, 2014. Surveillance and response: tools and approaches for the elimination stage of neglected tropical diseases. Acta Trop (in press; DOI: 10.1016/j.actatropica.2014.1009.1017).

Bi Y, Hu W, Yang H, Zhou XN, Yu W, Guo Y, Tong S, 2013.
Spatial patterns of malaria reported deaths in Yunnan province, China. Am J Trop Med Hyg 88, 526-535.

Bi Y, Tong S, 2014. Poverty and malaria in the Yunnan province, China. Infect Dis Poverty 3, 32.

Bowden J, Tierney JF, Copas AJ, Burdett S, 2011. Quantifying, displaying and accounting for heterogeneity in the meta-analysis of RCTs using standard and generalised Q statistics. BMC Med Res Methodol 11, 41.

Caminade C, Kovats S, Rocklov J, Tompkins AM, Morse AP, Colon-Gonzalez FJ, Stenlund H, Martens P, Lloyd SJ, 2014. Impact of climate change on global malaria distribution. Proc Natl Acad Sci U S A 111, 3286-3291.

Cao J, Sturrock HJ, Cotter C, Zhou S, Zhou H, Liu Y, Tang L, Gosling RD, Feachem RG, Gao Q, 2014. Communicating and monitoring surveillance and response activities for malaria elimination: China's “1-3-7” strategy. PLoS Med 11, e1001642.

Clements ACA, Reid HL, Kelly GC, Hay SI, 2013. Further shrinking the malaria map: how can geospatial science help to achieve malaria elimination? Lancet Infect Dis 13, 709-718.

Cotter C, Sturrock HJ, Hsiang MS, Liu J, Phillips AA, Hwang J, Gueye CS, Fullman N, Gosling RD, Feachem RG, 2013. The changing epidemiology of malaria elimination: new strategies for new challenges. Lancet 382, 900-911.

Diouf G, Kpanyen PN, Tokpa AF, Nie S, 2014. Changing landscape of malaria in China: progress and feasibility of malaria elimination. Asia Pac J Public Health 26, 93-100.

Elliott P, Wartenberg D, 2004. Spatial epidemiology: current approaches and future challenges. Environ Health Perspect 112, 998-1006.

Feachem RG, Phillips AA, Hwang J, Cotter C, Wielgosz B, Greenwood BM, Sabot O, Rodriguez MH, Abeyasinghe RR, Ghebreyesus TA et al., 2010. Shrinking the malaria map: progress and prospects. Lancet 376, 1566-1578.

Gething PW, Elyazar IR, Moyes CL, Smith DL, Battle KE, Guerra CA, Patil AP, Tatem AJ, Howes RE, Myers MF, 2012. A long neglected world malaria map: Plasmodium vivax endemicity in 2010. PLoS Negl Trop Dis 6, e1814.

Giardina F, Kasasa S, Sie A, Utzinger J, Tanner M, Vounatsou P, 2014. Effects of vector-control interventions on changes in risk of malaria parasitaemia in sub-Saharan Africa: a spatial and temporal analysis. Lancet Glob Health 2, e601-e615.

Huang F, Zhou S, Zhang S, Zhang H, Li W, 2011. Meteorological factors-based spatio-temporal mapping and predicting malaria in central China. Am J Trop Med Hyg 85, 560-567.

Li ZZ, Shang LY, Zhang L, Li DF, Sun YP, 1999. Control strategies of malaria in Henan province, China. Southeast Asian J Trop Med Public Health 30, 240-242.

Lu G, Zhou S, Horstick O, Wang X, Liu Y, Müller O, 2014. Malaria outbreaks in China (1990-2013): a systematic review. Malar J 13, 269. 
Mueller I, Galinski MR, Baird JK, Carlton JM, Kochar DK, Alonso PL, del Portillo HA, 2009. Key gaps in the knowledge of Plasmodium vivax, a neglected human malaria parasite. Lancet Infect Dis 9, 555-566.

Musa MI, Shohaimi S, Hashim NR, Krishnarajah I, 2012. A climate distribution model of malaria transmission in Sudan. Geospat Health 7, 27-36.

Noor AM, Kinyoki DK, Mundia CW, Kabaria CW, Mutua JW, Alegana VA, Fall IS, Snow RW, 2014. The changing risk of Plasmodium falciparum malaria infection in Africa: 2000-10: a spatial and temporal analysis of transmission intensity. Lancet 383, 1739-1747.

Ostfeld RS, Glass GE, Keesing F, 2005. Spatial epidemiology: an emerging (or re-emerging) discipline. Trends Ecol Evol 20, 328-336.

Reid H, Vallely A, Taleo G, Tatem AJ, Kelly G, Riley I, Harris I, Henri I, Iamaher S, Clements AC, 2010. Baseline spatial distribution of malaria prior to an elimination programme in Vanuatu. Malar J 9, 150.

Sleigh AC, Liu XL, Jackson S, Li P, Shang LY, 1998. Resurgence of vivax malaria in Henan province, China. Bull World Health Organ 76, 265-270.

Stresman G, Kobayashi T, Kamanga A, Thuma PE, Mharakurwa S, Moss WJ, Shiff C, 2012. Malaria research challenges in low prevalence settings. Malar J 11, 353.

Tambo E, Adedeji AA, Huang F, Chen JH, Zhou SS, Tang LH, 2012. Scaling up impact of malaria control programmes: a tale of events in sub-Saharan Africa and People's Republic of China. Infect Dis Poverty 1, 7.

Tang L, 2000. Progress in malaria control in China. Chin Med J (Engl) 113, 89-92.

Walker M, Winskill P, Basanez MG, Mwangangi JM, Mbogo C, Beier JC, Midega JT, 2013. Temporal and micro-spatial heterogeneity in the distribution of Anopheles vectors of malaria along the Kenyan coast. Parasit Vectors 6, 311.

Wang SQ, Li YC, Zhang ZM, Wang GZ, Hu XM, Qualls WA, Xue RD, 2014. Prevention measures and socio-economic development result in a decrease in malaria in Hainan, China. Malar J 13, 362.

Weiss DJ, Bhatt S, Mappin B, Van Boeckel TP, Smith DL, Hay SI, Gething PW, 2014. Air temperature suitability for
Plasmodium falciparum malaria transmission in Africa 20002012: a high-resolution spatiotemporal prediction. Malar J 13, 171.

WHO, 2012. World malaria report 2012. Geneva: World Health Organization.

Xu B, Li H, Webber RH, 1994. Malaria in Hubei province, China: approaching eradication. J Trop Med Hyg 97, 277281.

Yang GJ, Tanner M, Utzinger J, Malone JB, Bergquist R, Chan EY, Gao Q, Zhou XN, 2012. Malaria surveillance-response strategies in different transmission zones of the People's Republic of China: preparing for climate change. Malar J 11, 426.

Yang K, Li W, Sun LP, Huang YX, Zhang JF, Wu F, Hang DR, Steinmann P, Liang YS, 2013. Spatio-temporal analysis to identify determinants of Oncomelania hupensis infection with Schistosoma japonicum in Jiangsu province, China. Parasit Vectors 6, 138.

Yang K, Wang XH, Yang GJ, Wu XH, Qi YL, Li HJ, Zhou XN, 2008. An integrated approach to identify distribution of Oncomelania hupensis, the intermediate host of Schistosoma japonicum, in a mountainous region in China. Int J Parasitol 38, 1007-1016.

Yin JH, Yang MN, Zhou SS, Wang Y, Feng J, Xia ZG, 2013. Changing malaria transmission and implications in China towards national malaria elimination programme between 2010 and 2012. PLoS One 8, e74228.

Zheng Q, Vanderslott S, Jiang B, Xu LL, Liu CS, Huo LL, Duan LP, Wu NB, Li SZ, Xia ZG, et al., 2013. Research gaps for three main tropical diseases in the People's Republic of China. Infect Dis Poverty 2, 15.

Zhou SS, Huang F, Wang JJ, Zhang SS, Su YP, Tang LH, 2010. Geographical, meteorological and vectorial factors related to malaria re-emergence in Huang-Huai River of central China. Malar J 9, 337.

Zhou SS, Tang LH, Sheng HF, 2005. Malaria situation in the People's Republic of China in 2003. Chin J Parasitol Parasit Dis 23, 385-387 (in Chinese).

Zhou XN, Bergquist R, Tanner M, 2013. Elimination of tropical disease through surveillance and response. Infect Dis Poverty 2,1 . 